\title{
Multidimensional Linear and Nonlinear Partial Integro-Differential Equation in Bessel Potential Spaces with Applications in Option Pricing
}

\author{
Daniel Ševčovič * (1) and Cyril Izuchukwu Udeani (1) \\ Department of Applied Mathematics and Statistics, Comenius University in Bratislava, Mlynská Dolina, \\ 84248 Bratislava, Slovakia; cyril.izuchukwu@fmph.uniba.sk \\ * Correspondence: sevcovic@fmph.uniba.sk
}

check for

updates

Citation: Ševčovič, D.; Udeani, C.I. Multidimensional Linear and Nonlinear Partial Integro-Differential Equation in Bessel Potential Spaces with Applications in Option Pricing. Mathematics 2021, 9, 1463. https:// doi.org/10.3390/math9131463

Academic Editor: David Carfi

Received: 19 May 2021

Accepted: 19 June 2021

Published: 22 June 2021

Publisher's Note: MDPI stays neutral with regard to jurisdictional claims in published maps and institutional affiliations.

Copyright: (c) 2021 by the authors. Licensee MDPI, Basel, Switzerland. This article is an open access article distributed under the terms and conditions of the Creative Commons Attribution (CC BY) license (https:/ / creativecommons.org/licenses/by/ $4.0 /)$.

\begin{abstract}
The purpose of this paper is to analyze solutions of a non-local nonlinear partial integrodifferential equation (PIDE) in multidimensional spaces. Such class of PIDE often arises in financial modeling. We employ the theory of abstract semilinear parabolic equations in order to prove existence and uniqueness of solutions in the scale of Bessel potential spaces. We consider a wide class of Lévy measures satisfying suitable growth conditions near the origin and infinity. The novelty of the paper is the generalization of already known results in the one space dimension to the multidimensional case. We consider Black-Scholes models for option pricing on underlying assets following a Lévy stochastic process with jumps. As an application to option pricing in the one-dimensional space, we consider a general shift function arising from a nonlinear option pricing model taking into account a large trader stock-trading strategy. We prove existence and uniqueness of a solution to the nonlinear PIDE in which the shift function may depend on a prescribed large investor stock-trading strategy function.
\end{abstract}

Keywords: lévy measure; option pricing; strong kernel; hölder continuity; partial integro-differential equation; bessel potential spaces

MSC: 45K05; 35K58; 34G20; 91G20

\section{Introduction}

Our goal is to prove existence and uniqueness of a solution to a nonlinear parabolic partial integro-differential equation (PIDE) with the form:

$$
\begin{aligned}
\frac{\partial u}{\partial \tau}= & \Delta u+\int_{\mathbb{R}^{n}}\left[u(\tau, x+z)-u(\tau, x)-z \cdot \nabla_{x} u(\tau, x)\right] v(\mathrm{~d} z)+g\left(\tau, x, u, \nabla_{x} u\right), \\
& u(0, x)=u_{0}(x), \quad x \in \mathbb{R}^{n}, \tau \in(0, T)
\end{aligned}
$$

where $g$ is a given sufficiently smooth function. By $v$, we denote a positive measure on $\mathbb{R}^{n}$ such that its Radon derivative is a non-negative Lebesgue measurable function $h$ in $\mathbb{R}^{n}$, i.e., $v(\mathrm{~d} z)=h(z) \mathrm{d} z$.

Recently, the theory of stochastic processes have attracted significant attention owing to their important mathematical development and applications. Stochastic processes are mathematically rich objects with wide range of applications, e.g., in engineering, physics, and economics. It is worth noting that these processes occur in almost every discipline. Various stochastic processes, such as random walks, Markov chains, martingales, and Lévy processes have attracted significant attention due to their wide range of applications. In particular, the concept of Lévy measures plays a significant role owing to its theoretical developments and wealth of novel applications, e.g., to option pricing in financial mathematics. In the probability theory, the Laplace operator occurs as an infinitesimal generator of a stochastic process since it generates a semigroup corresponding to a symmetric stable process. The relationships between more general non-local operators and 
jump processes has been widely investigated. In particular, there is a relationship between the solution to PIDE and properties of the corresponding Markov jump process (c.f., Abels and Kassmann [1], or Florescu and Mariani [2]).

In recent decades, the role of PIDEs has been investigated in various fields, such as pure mathematical areas, biological sciences, and economics [2-4]. PIDE problems arising from financial mathematics, especially from option pricing models, have been of great interest to many researchers. In most cases, standard method for solving these problems lead to the study of parabolic equations. Mikulevičius and Pragaraustas [5] investigated solutions of the Cauchy problem to the parabolic PIDE with variable coefficients in Sobolev spaces. The results were applied in order to obtain solutions of the corresponding martingale problem. Crandal et al. [6] employed the notion of a viscosity solution to investigate the qualitative results. Their results were extended and generalized by Soner et al. [7] and Barles et al. [8] for the first and second order operators, respectively. Florescu and Mariani [2] used the Schaefer fixed point theorem to establish existence of a weak solution of the generalized PIDE. Amster et al. [9] used the method of upper and lower solutions. They proved the existence of solutions in a general domain for multiple assets and the regime switching jump-diffusion model. Cont et al. [10] investigated the actual connection between option pricing in exponential Lévy models and the corresponding PIDEs for European options and those with single or double barriers. They discussed the conditions for which prices of option are classical solution of the PIDE. Cruz and Ševčovič [11] employed the theory of abstract semilinear parabolic equations to obtain a solution to the nonlinear non-local PIDE (1) in the one-dimensional case. They employed the framework of a scale of Bessel potential spaces for general Lévy measures by considering classical Black-Scholes model that depends on various restricted assumptions, e.g., liquidity and completeness of the market. They relaxed the complete market hypothesis and assumed a Lévy process for underlying stock price to obtain a model for pricing European and American call and put options on an underlying asset characterized by a Lévy measure.

In this paper, we extend and generalize the results of Cruz and Ševčovič to a multidimensional space in the scale of Bessel potential Sobolev spaces. We shall also analyze the following generalization of PIDE:

$$
\frac{\partial u}{\partial \tau}=\frac{\sigma^{2}}{2} \Delta u+\int_{\mathbb{R}^{n}}\left[u(\tau, x+\xi)-u(\tau, x)-\xi \cdot \nabla_{x} u(\tau, x)\right] v(\mathrm{~d} z)+g\left(\tau, x, u, \nabla_{x} u\right) .
$$

where $\xi=\xi(\tau, x, z)$ is a shift function, which may depend on the variables $\tau>0, x, z \in \mathbb{R}$. An application for such a general shift function $\xi$ can be found in nonlinear option pricing models considering a large trader stock-trading strategy with the underlying asset price dynamic following the Lévy process (c.f., Cruz and Ševčovič [12]). If $\xi(x, z) \equiv z$, then (2) reduces to Equation (1). The nonlinearity $g$ often arises from applications occurring in pricing, e.g., XVA derivatives (c.f., Arregui et al. $[4,13]$ ) or applications of the penalty method for American option pricing under a PIDE model (c.f., Cruz and Ševčovič [11]).

Our aim is to investigate solutions to PIDE (1) in the framework of Bessel potential spaces for a multidimensional case, $n \geq 1$. They form a nested scale $\left\{X^{\gamma}\right\}_{\gamma \geq 0}$ of Banach spaces satisfying $X^{\gamma_{1}} \hookrightarrow X^{\gamma_{2}}$ for any $1 \geq \gamma_{1} \geq \gamma_{2} \geq 0$, and $X^{1} \equiv D(A), X^{0} \equiv X$. Here, the operator $A$ is sectorial in the space $X$ with a dense domain $D(A) \subset X$ (c.f., Henry [14]). An example of such an operator is the Laplace operator, i.e., $A=-\Delta$ in $\mathbb{R}^{n}$ with the domain $D(A) \equiv W^{2, p}\left(\mathbb{R}^{n}\right) \subset X \equiv L^{p}\left(\mathbb{R}^{n}\right)$. It is known that if $A=-\Delta$, then $X^{\gamma}$ is embedded in the Sobolev-Slobodecki space $W^{2 \gamma, p}\left(\mathbb{R}^{n}\right)$, which is a space consisting of all functions such that $2 \gamma$-fractional derivative belongs to the Lebesgue space $L^{p}\left(\mathbb{R}^{n}\right)$ of $p$-integrable functions (c.f., [14]). We investigate solutions to the PIDE (2) for a wide class of Lévy measures $v$ satisfying suitable growth conditions near $\pm \infty$ and origin in a higher-dimensional space.

The remainder of the paper is organized as follows. In Section 2, we present preliminaries and basic notions of Lévy measures, and introduce concept of admissible activity Lévy measures with some important examples. In Section 3, we establish the existence results to the nonlinear non-local PIDE (2) in the framework of Bessel potential spaces for 
the multidimensional case. We analyze the generalized nonlinear non-local PIDE (2), where the shift function $\xi=\xi(\tau, x, z)$ depends on the variables $x, z \in \mathbb{R}^{n}$. Section 4 presents an application of the proposed results in the one-dimensional space for pricing of options on underlying asset that follows Lévy processes. Section 5 presents the conclusions.

\section{Preliminaries, Definitions and Motivation}

In this section, we present some basic definitions of Lévy measures, and notion of admissible activity Lévy measures. In what follows, we denote the Euclidean norm in $\mathbb{R}^{n}$ and the norm in an infinite-dimensional function space (e.g., $\left.L^{p}\left(\mathbb{R}^{n}\right), X^{\gamma}\right)$ by $|\cdot|$, and $\|\cdot\|$, respectively. Henceforth, $a \cdot b$ stands for the usual Euclidean product in $\mathbb{R}^{n}$ with the norm $|z|=\sqrt{z \cdot z}$.

A Lévy process on $\mathbb{R}^{n}$ is a stochastic (right continuous) process $X=\left\{X_{t}, t \geq 0\right\}$ with the left limit with independent stationary increments. It is uniquely characterized by its Lévy exponent $\phi$ :

$$
\mathbb{E}_{x}\left(e^{i y \cdot X_{t}}\right)=e^{-t \phi(y)}, y \in \mathbb{R}^{n}
$$

The subscript $x$ in the expectation operator $\mathbb{E}_{x}$ indicates that the process $X_{t}$ starts from a given value $x$ at the origin $t=0$. The Lévy exponent $\phi$ has the following unique decomposition:

$$
\phi(y)=i b \cdot y+\sum_{i, j=1}^{n} a_{i j} y_{i} y_{j}+\int_{\mathbb{R}^{n}}\left(1-e^{i y \cdot z}+i y \cdot z 1_{|z| \leq 1}\right) v(\mathrm{~d} z),
$$

where $b \in \mathbb{R}^{n}$ is a constant vector, $\left(a_{i j}\right)$ is a constant matrix, which is positive semidefinite; $v(\mathrm{~d} z)$ is a non-negative measure on $\mathbb{R}^{n} \backslash\{0\}$ such that $\int_{\mathbb{R}^{n}} \min \left(1,|z|^{2}\right) v(\mathrm{~d} z)<\infty$ (c.f., [15]).

\section{Admissible Activity Lévy Measures}

Next, we recall notion of an admissible activity Lévy measure introduced by Cruz and Ševčovič $[11,12]$ for the one-dimensional case $n=1$. We shall extend this notion for the multidimensional case $n \geq 1$.

Definition 1. A measure $v$ in $\mathbb{R}^{n}$ is called an admissible activity Lévy measure if there exists a non-negative Lebesgue measurable function $h: \mathbb{R}^{n} \rightarrow \mathbb{R}$ such that $v(\mathrm{~d} z)=h(z) \mathrm{d} z$ such that

$$
0 \leq h(z) \leq C_{0}|z|^{-\alpha} e^{-D|z|-\mu|z|^{2}},
$$

for all $z \in \mathbb{R}^{n}$ and the shape parameters $\alpha, \mu \geq 0, D \in \mathbb{R},(D>0$ if $\mu=0)$. Here, $C_{0}>0$ is a positive constant.

It is worth noting that the class of admissible activity Lévy measures consists of various important measures often used in financial modeling of underlying stock dynamics with jumps. For instance, in the context of financial modeling, one of first jump-diffusion models was proposed by Merton [16]. Its Lévy measure has the form:

$$
v(\mathrm{~d} z)=\lambda \frac{1}{\left(2 \pi \delta^{2}\right)^{n / 2}} e^{-\frac{|z-m|^{2}}{2 \delta^{2}}} \mathrm{~d} z
$$

Here, the parameters $m \in \mathbb{R}^{n}, \lambda, \delta>0$, are given. Merton's measure is a finite activity Lévy measure, i.e., $v\left(\mathbb{R}^{n}\right)=\int_{\mathbb{R}^{n}} v(\mathrm{~d} z)<\infty$ with finite variation $\int_{|z| \leq 1}|z| v(\mathrm{~d} z)<\infty$.

A class of admissible Lévy measures also contains measures of the form:

$$
v(\mathrm{~d} z)=C_{0}|z|^{-\alpha} e^{-A|z|} \mathrm{d} z,
$$

where $\alpha \in \mathbb{R}, A>0$. Such a Lévy measure is of finite activity provided that $\alpha<n$, it has a finite variation if $\alpha<n+1$. 
In the one-dimensional case $n=1$, the class of admissible Lévy measures includes the double exponential model introduced by Kou [17], Variance Gamma model [18], Normal Inverse Gaussian (NIG), and CGMY models [19]. We refer the reader to the paper [11] by Cruz and Ševčovič for an overview of various examples of admissible Lévy measures in the one-dimensional case.

\section{Existence and Uniqueness Results}

In this section, we focus on the proof of the existence and uniqueness of a solution $u(\tau, x)$ to (2) for a wide class of Lévy measures. The PIDE (2) can be rewritten as follows:

$$
\frac{\partial u}{\partial \tau}+A u=f(u)+g\left(\tau, x, u, \nabla_{x} u\right), \quad u(0, x)=u_{0}(x), \quad x \in \mathbb{R}^{n}, \tau \in(0, T),
$$

where $A=-\left(\sigma^{2} / 2\right) \Delta$. The linear non-local operator $f$ is defined by:

$$
f(u)(\cdot)=\int_{\mathbb{R}^{n}}\left[u(\cdot+\xi)-u(\cdot)-\xi \cdot \nabla_{x} u(\cdot)\right] v(\mathrm{~d} z),
$$

where $\xi=\xi(\tau, x, z)$ is a given shift function. The mapping $g$ is assumed to be Hölder continuous in the $\tau$ variable and Lipschitz continuous in the remaining variables. The proof of the existence, continuation, and uniqueness of a solution is based on the abstract theory of semilinear parabolic equations according to Henry [14]. A solution to the PIDE (6) is constructed in the scale of the Bessel potential spaces $\mathscr{L}_{2 \gamma}^{p}\left(\mathbb{R}^{n}\right), \gamma \geq 0$, for the multidimensional case, $n \geq 1$. These spaces can be considered to be a natural extension of the classical Sobolev spaces $W^{k, p}\left(\mathbb{R}^{n}\right)$ for non-integer values of order $k$. The nested scale of Bessel potential spaces allows for a finer formulation of existence and uniqueness results compared to the classical Sobolev spaces.

In what follows, we briefly recall the construction and basic properties of Bessel potential spaces. Recall that if $A$ is a sectorial operator in a Banach space $X$, then $-A$ is a generator of an analytic semigroup $\left\{e^{-A t}, t \geq 0\right\}$ acting on $X$ (c.f., [14] Chapter I). For any $\gamma>0$, we can introduce the operator $A^{-\gamma}: X \rightarrow X$ as follows: $A^{-\gamma}=\frac{1}{\Gamma(\gamma)} \int_{0}^{\infty} \xi^{\gamma-1} e^{-A \xi} \mathrm{d} \xi$. Then, the fractional power space $X^{\gamma}=D\left(A^{\gamma}\right)$ is the domain of the operator $A^{\gamma}=\left(A^{-\gamma}\right)^{-1}$, i.e., $X^{\gamma}=$ $\left\{u \in X: \exists \varphi \in X, u=A^{-\gamma} \varphi\right\}$. The norm is defined as follows: $\|u\|_{X^{\gamma}}=\left\|A^{\gamma} u\right\|_{X}=\|\varphi\|_{X}$. Furthermore, we have continuous embedding: $D(A) \equiv X^{1} \hookrightarrow X^{\gamma_{1}} \hookrightarrow X^{\gamma_{2}} \hookrightarrow X^{0} \equiv X$, for $0 \leq \gamma_{2} \leq \gamma_{1} \leq 1$.

Let us recall the convolution operator $(G * \varphi)(x)=\int_{\mathbb{R}^{n}} G(x-y) \varphi(y) \mathrm{d} y$. According to ([14] Section 1.6), $A=-\left(\sigma^{2} / 2\right) \Delta$ is a sectorial operator in the Lebesgue space $X=L^{p}\left(\mathbb{R}^{n}\right)$ for any $p \geq 1, n \geq 1$, and $D(A) \subset W^{2, p}\left(\mathbb{R}^{n}\right)$. Now, it follows from ([20] Chapter 5$)$ that the space $X^{\gamma}, \gamma>0$, can be identified with the Bessel potential space $\mathscr{L}_{2 \gamma}^{p}\left(\mathbb{R}^{n}\right)$, where

$$
\mathscr{L}_{2 \gamma}^{p}\left(\mathbb{R}^{n}\right):=\left\{u \in X: \exists \varphi \in X, u=G_{2 \gamma} * \varphi\right\} .
$$

Here, $G_{2 \gamma}$ is the Bessel potential function,

$$
G_{2 \gamma}(x)=\frac{1}{(4 \pi)^{n / 2} \Gamma(\gamma)} \int_{0}^{\infty} y^{-1+\gamma-n / 2} e^{-\left(y+|x|^{2} /(4 y)\right)} \mathrm{d} y .
$$

The norm of $u=G_{2 \gamma} * \varphi$ is given by $\|u\|_{X^{\gamma}}=\|\varphi\|_{L^{p}}$. The space $X^{\gamma}$ is continuously embedded in the fractional Sobolev-Slobodeckii space $W^{2 \gamma, p}\left(\mathbb{R}^{n}\right)$ (c.f., [14] Section 1.6).

By $C_{0}>0$, we shall denote a generic constant which is independent of the solution $u$; however, it may depend on model parameters, e.g., $n \geq 1, p \geq 1, \gamma \in[0,1)$.

Proposition 1. Let us define the mapping $Q(u, \xi)$ as follows:

$$
Q(u, \xi)=u(x+\xi(x))-\xi(x) \cdot \nabla_{x} u(x), \quad x \in \mathbb{R}^{n}
$$


Then, there exists a constant $\hat{C}>0$ such that for any vector valued functions $\xi_{1}, \xi_{2} \in\left(L^{\infty}\left(\mathbb{R}^{n}\right)\right)^{n}$, and $u$ such that $\nabla_{x} u \in\left(X^{\gamma-1 / 2}\right)^{n}, 1 / 2 \leq \gamma<1$, the following estimate holds:

$$
\left\|Q\left(u, \xi_{1}\right)-Q\left(u, \xi_{2}\right)\right\|_{L^{p}\left(\mathbb{R}^{n}\right)} \leq \hat{C}\left\|\xi_{1}-\xi_{2}\right\|_{\infty}^{2 \gamma-1}\left(\left\|\xi_{1}\right\|_{\infty}+\left\|\xi_{2}\right\|_{\infty}\right)\left\|\nabla_{x} u\right\|_{X^{\gamma-1 / 2}} .
$$

Proof. Let $u \in X$ be such that $\nabla_{x} u \in\left(X^{\gamma-1 / 2}\right)^{n}$, i.e., $\partial_{x_{i}} u \in X^{\gamma-1 / 2}$ for each $i=1, \cdots, n$. Then $\nabla_{x} u=A^{-(2 \gamma-1) / 2} \varphi=G_{2 \gamma-1} * \varphi$ for some $\varphi \in\left(L^{p}\left(\mathbb{R}^{n}\right)\right)^{n}$, and $\left\|\nabla_{x} u\right\|_{X^{\gamma-1 / 2}}=$ $\left\|A^{(2 \gamma-1) / 2} \nabla_{x} u\right\|_{X}=\|\varphi\|_{L^{p}}$. Here, $\varphi=\left(\varphi_{1}, \cdots, \varphi_{n}\right)^{T}$ and $\partial_{x_{i}} u=G_{2 \gamma-1} * \varphi_{i}$. Let $x, \xi \in \mathbb{R}^{n}$. Then

$$
\nabla_{x} u(x+\xi)=G_{2 \gamma-1}(x+\xi-\cdot) * \varphi(\cdot), \quad \nabla_{x} u(x)=G_{2 \gamma-1}(x-\cdot) * \varphi(\cdot) .
$$

Recall that the convolution operator satisfies the following inequality:

$$
\|\psi * \varphi\|_{L^{p}\left(\mathbb{R}^{n}\right)} \leq\|\psi\|_{L^{q}\left(\mathbb{R}^{n}\right)}\|\varphi\|_{L^{r}\left(\mathbb{R}^{n}\right)},
$$

where $p, q, r \geq 1$ and $1 / p+1=1 / q+1 / r$ (see [14] Section 1.6). In particular, for $q=1$ we have $\|\psi * \varphi\|_{L^{p}} \leq\|\psi\|_{L^{1}}\|\varphi\|_{L^{p}}$. For the modulus of continuity of the Bessel potential function $G_{\alpha}, \alpha \in(0,1)$, the following estimate holds:

$$
\left\|G_{\alpha}(\cdot+h)-G_{\alpha}(\cdot)\right\|_{L^{1}} \leq C_{0}|h|^{\alpha},
$$

for any $h \in \mathbb{R}^{n}$ (c.f., [20] Chapter 5.4, Proposition 7). Let $\xi_{1}, \xi_{2}$ be bounded vector valued functions, i.e., $\xi_{1}, \xi_{2} \in\left(L^{\infty}\left(\mathbb{R}^{n}\right)\right)^{n}$. Then, for any $x \in \mathbb{R}^{n}$ and $\theta \in[0,1]$, we have

$$
\begin{aligned}
& u\left(x+\xi_{1}(x)\right)-u\left(x+\xi_{2}(x)\right)-\left(\xi_{1}(x)-\xi_{2}(x)\right) \cdot \nabla_{x} u(x) \\
= & u\left(x+\xi_{1}(x)\right)-\nabla_{x} u(x)+\xi_{1}(x) \cdot \nabla_{x} u(x) \\
& -\left[u\left(x+\xi_{2}(x)\right)-\nabla_{x} u(x)-\xi_{2}(x) \cdot \nabla_{x} u(x)\right] \\
= & \left(\xi_{1}(x)-\xi_{2}(x)\right) \int_{0}^{1} \nabla_{x} u\left(x+\xi_{1}(x)\right)-\nabla_{x} u(x) \mathrm{d} \theta \\
& +\int_{0}^{1} \nabla_{x} u\left(x+\theta \xi_{1}(x)\right)-\nabla_{x} u\left(x+\theta \xi_{2}(x)\right) \mathrm{d} \theta .
\end{aligned}
$$

Now,

$$
\begin{aligned}
& \left\|Q\left(u, \xi_{1}\right)-Q\left(u, \xi_{2}\right)\right\|_{L^{p}\left(\mathbb{R}^{n}\right)}^{p} \\
= & \int_{\mathbb{R}^{n}}\left|u\left(x+\xi_{1}(x)\right)-u\left(x+\xi_{2}(x)\right)-\left(\xi_{1}(x)-\xi_{2}(x)\right) \cdot \nabla_{x} u(x)\right|^{p} \mathrm{~d} x \\
\leq & \int_{\mathbb{R}^{n}}\left|\left(\xi_{1}(x)-\xi_{2}(x)\right) \int_{0}^{1} \nabla_{x} u\left(x+\theta \xi_{1}(x)\right)-\nabla_{x} u(x) \mathrm{d} \theta\right|^{p} \mathrm{~d} x \\
& +\int_{\mathbb{R}^{n}}\left|\xi_{2}(x) \int_{0}^{1} \nabla_{x} u\left(x+\theta \xi_{1}(x)\right)-\nabla_{x} u\left(x+\theta \xi_{2}(x)\right) \mathrm{d} \theta\right|^{p} \mathrm{~d} x \\
\leq & \left\|\xi_{1}-\xi_{2}\right\|_{\infty}^{p} \int_{0}^{1} \int_{\mathbb{R}^{n}}\left|\nabla_{x} u\left(x+\theta \xi_{1}(x)\right)-\nabla_{x} u(x)\right|^{p} d x d \theta \\
& +\left\|\xi_{2}\right\|_{\infty}^{p} \int_{0}^{1} \int_{\mathbb{R}^{n}} \mid \nabla_{x} u\left(x+\theta \xi_{1}(x)\right)-\nabla_{x} u\left(x+\left.\theta \xi_{2}(x)\right|^{p} d x d \theta\right. \\
\leq & \left\|\xi_{1}-\xi_{2}\right\|_{\infty}^{p} \int_{0}^{1}\left\|\left(G_{2 \gamma-1}\left(\cdot+\theta \xi_{1}\right)-G_{2 \gamma-1}(\cdot)\right) * \varphi\right\|_{L^{p}}^{p} d \theta \\
& +\left\|\xi_{2}\right\|_{\infty}^{p} \int_{0}^{1}\left\|\left(G_{2 \gamma-1}\left(\cdot+\theta \xi_{1}\right)-G_{2 \gamma-1}\left(\cdot+\theta \xi_{2}\right)\right) * \varphi\right\|_{L^{p}}^{p} d \theta \\
\leq & \left\|\xi_{1}-\xi_{2}\right\|_{\infty}^{p} \int_{0}^{1}\left\|G_{2 \gamma-1}\left(\cdot+\theta \xi_{1}\right)-G_{2 \gamma-1}(\cdot)\right\|_{L^{1}}^{p}\|d \theta \varphi\|_{L^{p}}^{p} \\
& +\left\|\xi_{2}\right\|_{\infty}^{p} \int_{0}^{1}\left\|G_{2 \gamma-1}\left(\cdot+\theta \xi_{1}\right)-G_{2 \gamma-1}\left(\cdot+\theta \xi_{2}\right)\right\|_{L^{1}}^{p} d \theta\|\varphi\|_{L^{p}}^{p} \\
\leq & \left(\left\|\xi_{1}-\xi_{2}\right\|_{\infty}^{p}\left\|\xi_{1}\right\|_{\infty}^{(2 \gamma-1) p}+\left\|\xi_{2}\right\|_{\infty}^{p}\left\|\xi_{1}-\xi_{2}\right\|_{\infty}^{(2 \gamma-1) p}\right) C_{0}^{p}\left\|\nabla_{x} u\right\|_{X^{\gamma}-1 / 2}^{p} \\
\leq & \left\|\xi_{1}-\xi_{2}\right\|_{\infty}^{(2 \gamma-1) p}\left(\left\|\xi_{1}\right\|_{\infty}^{p}+\left\|\xi_{2}\right\|_{\infty}^{(2-2 \gamma) p}\left\|\xi_{1}\right\|_{\infty}^{(2 \gamma-1) p}+\left\|\xi_{2}\right\|_{\infty}^{p}\right) C_{0}^{p}\left\|\nabla_{x} u\right\|_{X^{\gamma-1} / 2}^{p} .
\end{aligned}
$$

By Young's inequality, we have $a b \leq \frac{a^{\alpha}}{\alpha}+\frac{b^{\beta}}{\beta}$ for any $a, b \geq 0$, and $\alpha, \beta>1$ with $1 / \alpha+$ $1 / \beta=1$ (c.f., [21]). Let us set $\alpha=1 /(2-2 \gamma), \beta=1 /(2 \gamma-1)$. Then, $1 / \alpha+1 / \beta=1$ and 
we obtain $\left\|\xi_{2}\right\|_{\infty}^{(2-2 \gamma) p}\left\|\xi_{1}\right\|_{\infty}^{(2 \gamma-1) p} \leq(2-2 \gamma)\left\|\xi_{2}\right\|_{\infty}^{p}+(2 \gamma-1)\left\|\xi_{1}\right\|_{\infty}^{p} \leq 2\left\|\xi_{2}\right\|_{\infty}^{p}+\left\|\xi_{1}\right\|_{\infty}^{p}$. Therefore,

$$
\begin{aligned}
\left\|Q\left(u, \xi_{1}\right)-Q\left(u, \xi_{2}\right)\right\|_{L^{p}\left(\mathbb{R}^{n}\right)}^{p} & \leq 2\left\|\xi_{1}-\xi_{2}\right\|_{\infty}^{(2 \gamma-1) p}\left(\left\|\xi_{1}\right\|_{\infty}^{p}+\left\|\xi_{2}\right\|_{\infty}^{p}\right) C_{0}^{p}\left\|\nabla_{x} u\right\|_{X^{\gamma-1 / 2}}^{p} \\
& \leq 2 C_{0}^{p}\left\|\xi_{1}-\xi_{2}\right\|_{\infty}^{(2 \gamma-1) p}\left(\left\|\xi_{1}\right\|_{\infty}+\left\|\xi_{2}\right\|_{\infty}\right)^{p}\left\|\nabla_{x} u\right\|_{X^{\gamma-1 / 2}}^{p} .
\end{aligned}
$$

Hence, the pointwise estimate holds with the constant $\hat{C}=2^{1 / p} C_{0}>0$.

Applying Proposition 1 with $\xi_{1}=\xi$ and $\xi_{2}=0$, we obtain the following corollary.

Corollary 1. Let $u$ be such that $\nabla_{x} u \in\left(X^{\gamma-1 / 2}\right)^{n}$ where $1>\gamma \geq 1 / 2$. Then, for any $\xi \in \mathbb{R}^{n}$, the following pointwise estimate holds:

$$
\|Q(u, \xi)\|_{L^{p}\left(\mathbb{R}^{n}\right)} \leq C_{0}|\xi|^{2 \gamma}\left\|\nabla_{x} u\right\|_{X^{\gamma-1 / 2}} .
$$

Next, we consider the case when the non-local integral term depends on $x$ and $z$ variables. It is a generalization of the result ([12], Lemma 3.4) due to Cruz and Ševčovič proven for the case where $\xi(x, z) \equiv z$.

Proposition 2. Suppose that the shift mapping $\xi=\xi(x, z)$ satisfies $\sup _{x \in \mathbb{R}}|\xi(x, z)| \leq C_{0}|z|^{\omega}(1+$ $\left.e^{D_{0}|z|}\right)$ for some constants $C_{0}>0, D_{0} \geq 0, \omega>0$ and any $z \in \mathbb{R}$. Assume $v$ is a Léry measure with the shape parameters $\alpha, D$, and either $\mu>0, D \in \mathbb{R}$, or $\mu=0$ and $D>D_{0} \geq 0$. Assume $1 / 2 \leq \gamma<1$, and $\gamma>(\alpha-n) /(2 \omega)$. Then there exists a constant $C_{0}>0$ such that

$$
\|f(u)\|_{L^{p}} \leq C_{0}\left\|\nabla_{x} u\right\|_{X^{\gamma-1 / 2}}
$$

provided that $\nabla_{x} u \in\left(X^{\gamma-1 / 2}\right)^{n}$. If $u \in X^{\gamma}$ then $\|f(u)\|_{L^{p}} \leq C\|u\|_{X^{\gamma}}$, i.e., $f: X^{\gamma} \rightarrow X$ is a bounded linear operator.

Proof. The Lévy measure $v(\mathrm{~d} z)$ is given by $v(\mathrm{~d} z)=h(z) \mathrm{d} z$. Let us denote the auxiliary function $\tilde{h}(z)=|z|^{\alpha} h(z)$. Then, $0 \leq \tilde{h}(z) \leq C_{0} e^{-D|z|-\mu|z|^{2}}$. Since $h(z)=|z|^{-\alpha} \tilde{h}(z)=$ $h_{1}(z) h_{2}(z)$, where $h_{1}(z)=|z|^{-\beta} \tilde{h}(z)^{\frac{1}{2}}$ and $h_{2}(z)=|z|^{\beta-\alpha} \tilde{h}(z)^{\frac{1}{2}}$. Applying Proposition 1 with $\xi_{1}=\xi_{1} \xi_{2}=0$, and using the Hölder inequality, we obtain

$$
\begin{aligned}
& \|f(u)\|_{L^{p}}^{p}=\int_{\mathbb{R}^{n}}\left|\int_{\mathbb{R}^{n}}\left(u(x+\xi(x, z))-u(x)-\xi(x, z) \cdot \nabla_{x} u(x)\right) h(z) \mathrm{d} z\right|^{p} \mathrm{~d} x \\
& \leq \int_{\mathbb{R}^{n}} \int_{\mathbb{R}^{n}}\left|u(x+\xi(x, z))-u(x)-\xi(x, z) \cdot \nabla_{x} u(x)\right|^{p} h_{1}(z)^{p} \mathrm{~d} z \\
& \times\left(\int_{\mathbb{R}^{n}} h_{2}(z)^{q} \mathrm{~d} z\right)^{p / q} \mathrm{~d} x \\
& =\int_{\mathbb{R}^{n}}\left(\int_{\mathbb{R}^{n}}\left|u(x+\xi(x, z))-u(x)-\xi(x, z) \cdot \nabla_{x} u(x)\right|^{p} \mathrm{~d} x\right) h_{1}(z)^{p} \mathrm{~d} z \\
& \times\left(\int_{\mathbb{R}^{n}} h_{2}(z)^{q} \mathrm{~d} z\right)^{p / q} \\
& \leq C_{0}^{p}\left\|\nabla_{x} u\right\|_{X^{\gamma-1 / 2}}^{p} \int_{\mathbb{R}^{n}}|\xi(x, z)|^{2 \gamma p}|z|^{-\beta p} \tilde{h}(z)^{p / 2} \mathrm{~d} z\left(\int_{\mathbb{R}^{n}} h_{2}(z)^{q} \mathrm{~d} z\right)^{p / q} \\
& \leq C_{0}^{p}\left\|\nabla_{x} u\right\|_{X^{\gamma-1 / 2}}^{p} \int_{\mathbb{R}^{n}}|z|^{(2 \gamma \omega-\beta) p} \tilde{h}(z)^{p / 2} \mathrm{~d} z\left(\int_{\mathbb{R}^{n}} h_{2}(z)^{q} \mathrm{~d} z\right)^{p / q} .
\end{aligned}
$$

Assuming $p, q \geq 1,1 / p+1 / q=1$ are such that

$$
(2 \gamma \omega-\beta) p>-n, \quad(\beta-\alpha) q=(\beta-\alpha) \frac{p}{p-1}>-n,
$$


then, the integrals $\int_{\mathbb{R}^{n}}|z|^{(2 \gamma \omega-\beta) p} \tilde{h}(z)^{p / 2} \mathrm{~d} z$ and $\int_{\mathbb{R}^{n}} h_{2}(z)^{q} \mathrm{~d} z=\int_{\mathbb{R}^{n}}|z|^{(\beta-\alpha) q} \tilde{h}(z)^{q / 2} \mathrm{~d} z$ are finite, provided that the shape parameters satisfy either $\mu>0, D \in \mathbb{R}$, or $\mu=0, D>D_{0} \geq$ 0 . As $\gamma>(\alpha-n) /(2 \omega)$, there exists $\beta>1$ satisfying

$$
\alpha-n+n / p<\beta<2 \gamma \omega+n / p .
$$

Therefore, there exists $C_{0}>0$ such that $\|f(u)\|_{L^{p}} \leq C_{0}\left\|\nabla_{x} u\right\|_{X^{\gamma-1 / 2}}$.

Let $C\left([0, T], X^{\gamma}\right)$ denote the Banach space consisting of continuous functions from $[0, T]$ to $X^{\gamma}$ with the maximum norm. The following proposition is due to Henry [14] (see also Cruz and Ševčovič [11]).

Proposition 3. Refs. [11,14] Proposition 3.5 Suppose that the linear operator $-A$ is a generator of an analytic semigroup $\left\{e^{-A t}, t \geq 0\right\}$ in a Banach space $X$. Assume the initial condition $U_{0}$ belongs to the space $X^{\gamma}$ where $0 \leq \gamma<1$. Suppose that the mappings $F:[0, T] \times X^{\gamma} \rightarrow X$ and $h:(0, T] \rightarrow X$ are Hölder continuous in the $\tau$ variable, $\int_{0}^{T}\|h(\tau)\|_{X} \mathrm{~d} \tau<\infty$, and F is Lipschitz continuous in the $U$ variable. Then, for any $T>0$, there exists a unique solution the abstract semilinear evolution equation: $\partial_{\tau} U+A U=F(\tau, U)+h(\tau)$ such that $U \in C\left([0, T], X^{\gamma}\right), U(0)=$ $U_{0}, \partial_{\tau} U(\tau) \in X, U(\tau) \in D(A)$ for any $\tau \in(0, T)$. The function $U$ is a solution in the mild (integral) sense, i.e., $U(\tau)=e^{-A \tau} U_{0}+\int_{0}^{\tau} e^{-A(\tau-s)}(F(s, U(s))+h(s)) \mathrm{d} s, \tau \in[0, T]$.

Applying Propositions 2 and 3, we can state the following result which is a nontrivial generalization of the result shown by Ševčovič and Cruz [11] in the one space dimension $n=1$.

Theorem 1. Suppose that the shift mapping $\xi=\xi(x, z)$ satisfies $\sup _{x \in \mathbb{R}}|\xi(x, z)| \leq C_{0}|z|^{\omega}(1+$ $\left.e^{D_{0}|z|}\right), z \in \mathbb{R}^{n}$, for some constants $C_{0}>0, D_{0} \geq 0, \omega>0$. Assume $v$ is an admissible activity Lévy measure with the shape parameters $\alpha, D$, and, either $\mu>0, D \in \mathbb{R}$, or $\mu=0$ and $D>D_{0} \geq 0$. Assume $1 / 2 \leq \gamma<1$ and $\gamma>(\alpha-n) /(2 \omega), n \geq 1$. Suppose that $g\left(\tau, x, u, \nabla_{x} u\right)$ is Hölder continuous in the $\tau$ variable and Lipschitz continuous in the remaining variables, respectively. Assume $u_{0} \in X^{\gamma}$, and $T>0$. Then, there exists a unique mild solution $u$ to PIDE (2) satisfying $u \in C\left([0, T], X^{\gamma}\right)$.

\section{Applications to Option Pricing}

The classical linear Black-Scholes model and its multidimensional generalizations have been widely used in the analysis of financial markets. According to this model, the price $V=V(t, S)$ of an option on an underlying asset price $S$ at time $t \in[0, T]$ can be obtained from a solution to the linear Black-Scholes parabolic equation:

$$
\frac{\partial V}{\partial t}+\frac{1}{2} \sigma^{2} S^{2} \frac{\partial^{2} V}{\partial S^{2}}+r S \frac{\partial V}{\partial S}-r V=0, \quad V(T, S)=\Phi(S),
$$

$t \in[0, T), S>0$. The parameter $r>0$ represents the risk-free interest rate of a bond, $\sigma>0$ is the historical volatility of the underlying asset price $S$ time series. The underlying asset price is assumed to follow the geometric Brownian motion $d S / S=\mu d t+\sigma d W_{t}$. Here, $\left\{W_{t}, t \geq 0\right\}$ represents the standard Wiener process. The terminal condition $\Phi(S)$ represents the pay-off diagram at maturity $t=T, \Phi(S)=(S-K)^{+}$(call option case) or $\Phi(S)=(K-S)^{+}$(put option case).

In the multidimensional case, where the option price $V\left(t, S_{1}, \cdots, S_{n}\right)$ depends on the vector of $n$ underlying stochastic assets $S=\left(S_{1}, \cdots, S_{n}\right)$ with the volatilities $\sigma_{i}$ and mutual correlations $\varrho_{i j}, i, j=1, \cdots, n$, the Black-Scholes pricing equation can be written in the form:

$$
\frac{\partial V}{\partial t}+\frac{1}{2} \sum_{i=1}^{n} \sum_{j=1}^{n} \rho_{i j} \sigma_{i} \sigma_{j} S_{i} S_{j} \frac{\partial^{2} V}{\partial S_{i} \partial S_{j}}+r \sum_{i=1}^{n} S_{i} \frac{\partial V}{\partial S_{i}}-r V=0, \quad V(T, S)=\Phi(S) .
$$


Both Equations (8) and (9) can be transformed into Equation (1) defined on the whole space $\mathbb{R}^{n}$ (c.f., Ševčovič, Stehlíková, Mikula [22] Chapter 4, Section 5).

However, stock market observations indicate that the models (8) and (9) were derived under several restrictive assumptions, such as perfect replication of a portfolio and its liquidity, completeness and frictionless of the financial market, and/or absence of transaction costs. However, these assumptions are often violated. In recent decades, effects of nontrivial transaction costs were investigated by Leland [23], Kwok [24], Avellaneda and Paras [25], Ševčovič and Žitňanská [26], and many other authors. Feedback and illiquid market effects due to large traders choosing given stock-trading strategies were investigated by Schönbucher and Willmott [27], Frey and Patie [28], Frey and Stremme [29]. Effects of the risk arising from an unprotected portfolio were analyzed by Jandačka and Ševčovič [30]. Option pricing models based on utility maximization were analyzed by Barles and Soner [31]. A common feature of these generalizations of the linear Black-Scholes Equation (8) is that the constant volatility $\sigma$ is replaced by a nonlinear function depending on the second derivative $\partial_{S}^{2} V$ of the option price $V$. Among nonlinear generalizations of the Black-Scholes equation, there is the nonlinear Black-Scholes model derived by Frey and Stremme [30] (see also [28,32]). The underlying asset dynamics takes into account the presence of feedback effects due to influence of a large trader choosing particular stock-trading strategy (see also [27]).

In a recent paper [12], Cruz and Ševčovič generalized the Black-Scholes equation in two important directions. First, following the ideas Frey and Stremme [30], the model incoporates the effect of a large trader. Secondly, Cruz and Ševčovič relaxed the assumption on liquidity of market by assuming that the underlying asset price follows a Lévy stochastic process with jumps. The resulting governing equation is the following nonlinear PIDE:

$$
\begin{aligned}
\frac{\partial V}{\partial t} & +\frac{1}{2} \frac{\sigma^{2} S^{2}}{\left(1-\varrho S \partial_{S} \phi\right)^{2}} \frac{\partial^{2} V}{\partial S^{2}}+r S \frac{\partial V}{\partial S}-r V \\
& +\int_{\mathbb{R}}\left(V(t, S+H)-V(t, S)-H \frac{\partial V}{\partial S}\right) v(\mathrm{~d} z)=0
\end{aligned}
$$

where the shift function $H=H(\phi, S, z)$ depends on the large investor stock-trading strategy function $\phi=\phi(t, S)$ as a solution to the following implicit algebraic equation:

$$
H=\rho S(\phi(t, S+H)-\phi(t, S))+S\left(e^{z}-1\right) .
$$

The large trader strategy function $\phi$ may depend on the derivative $\partial_{S} V$ of the option price $V$, e.g., $\phi(t, S)=\partial_{S} V(t, S)$. Nevertheless, in our application, we assume the trading strategy function $\phi(t, S)$ is prescribed and it is globally Hölder continuous.

If $\rho=0$, then $H=S\left(e^{z}-1\right)$. Using the standard transformation $\tau=T-t, x=\ln \left(\frac{S}{K}\right)$ and setting $V(t, S)=e^{-r \tau} u(\tau, x)$, then Equation (10) can be reduced to a linear PIDE of the form (1) in the one-dimensional space $(n=1)$.

If $\rho>0$, then (10) can be transformed into a nonlinear parabolic PIDE. Indeed, suppose that the transformed large trader stock-trading strategy $\psi(\tau, x)=\phi(t, S)$. Then, $V(t, S)$ solves Equation (10) if and only if the transformed function $u(\tau, x)$ is a solution to the nonlinear parabolic equation:

$$
\begin{aligned}
\frac{\partial u}{\partial \tau} & =\frac{\sigma^{2}}{2} \frac{1}{\left(1-\rho \partial_{x} \psi\right)^{2}} \frac{\partial^{2} u}{\partial^{2} x}+\left(r-\frac{\sigma^{2}}{2} \frac{1}{\left(1-\rho \partial_{x} \psi\right)^{2}}-\delta(\tau, x)\right) \frac{\partial u}{\partial x} \\
& +\int_{\mathbb{R}}\left(u(\tau, x+\xi)-u(\tau, x)-\xi \frac{\partial u}{\partial x}(\tau, x)\right) v(\mathrm{~d} z), \quad u(0, x)=\Phi\left(K e^{x}\right),
\end{aligned}
$$

$\tau \in[0, T], x \in \mathbb{R}$. The shift function $\xi(\tau, x, z)$ is a solution to the algebraic equation:

$$
e^{\xi}=e^{z}+\rho(\psi(\tau, x+\xi)-\psi(\tau, x))
$$


and $\delta(\tau, x)=\int_{\mathbb{R}}\left(e^{\xi}-1-\xi\right) v(\mathrm{~d} z)=\int_{\mathbb{R}}\left(e^{z}-1-\xi+\rho(\psi(\tau, x+\xi)-\psi(\tau, x))\right) v(\mathrm{~d} z)$. For small values of $0<\rho \ll 1$, we can construct the first order asymptotic expansion $\xi(\tau, x, z)=\xi_{0}(\tau, x, z)+\rho \xi_{1}(\tau, x, z)$. For $\rho=0$, we obtain $\xi_{0}(\tau, x, z)=z$. Hence

$$
e^{z+\rho \xi_{1}}=e^{z}+\rho\left(\psi\left(\tau, x+z+\rho \xi_{1}\right)-\psi(\tau, x)\right) .
$$

Taking the first derivative of the above implicit equation with respect to $\rho$ and evaluating it at the origin $\rho=0$, we obtain $\xi_{1}=e^{-z}(\psi(\tau, x+z)-\psi(\tau, x))$, i.e.,

$$
\xi(\tau, x, z)=z+\rho e^{-z}(\psi(\tau, x+z)-\psi(\tau, x)) .
$$

As a consequence, we obtain the following lemma.

Lemma 1. Assume that the stock-trading strategy $\phi=\phi(t, S)$ is a globally $\omega$-Hölder continuous function, $0<\omega \leq 1$. Then, the transformed function $\psi(\tau, x)=\phi(t, S)$ is $\omega$-Hölder continuous, and the first order asymptotic expansion $\xi(\tau, x, z)$ of the nonlinear algebraic Equation (13) is $\omega$-Hölder continuous in all variables. Furthermore, there exists a constant $C_{0}>0$ such that $\sup _{\tau, x}|\xi(\tau, x, z)| \leq C_{0}|z|^{\omega}\left(1+e^{|z|}\right)$ for any $z \in \mathbb{R}$.

In what follows, we shall consider a simplified linear approximation of (10) where we set $\rho=0$ in the diffusion function, but we keep the shift function $H$ depending on the parameter $\rho$. Then, the transformed Cauchy problem for the solution $u$ with the first order approximation of the shift function $\xi$ reads as follows:

$$
\begin{aligned}
\frac{\partial u}{\partial \tau}= & \frac{\sigma^{2}}{2} \frac{\partial^{2} u}{\partial^{2} x}+\left(r-\frac{\sigma^{2}}{2}+\delta(\tau, x)\right) \frac{\partial u}{\partial x} \\
& +\int_{\mathbb{R}}\left(u(\tau, x+\xi)-u(\tau, x)-\xi \frac{\partial u}{\partial x}(\tau, x)\right) v(\mathrm{~d} z),
\end{aligned}
$$

$\tau \in[0, T], x \in \mathbb{R}$, where $\xi(\tau, x, z)=z+\rho(\psi(\tau, x+z))-\psi(\tau, x))$.

Note that the call/put option pay-off functions $\Phi(S)=\Phi\left(K e^{x}\right)=(S-K)^{+}=$ $K\left(e^{x}-1\right)^{+} / \Phi(S)=\Phi\left(K e^{x}\right)=(K-S)^{+}=K\left(1-e^{x}\right)^{+}$do not belong to the Banach space $X^{\gamma}$. Following [11], the procedure on how to overcome this problem and formulate existence and uniqueness of a solution to the PIDE (15) is based on shifting the solution $u$ by $u^{B S}$. Here, $u^{B S}(\tau, x)=e^{r \tau} V^{B S}\left(T-\tau, K e^{x}\right)$ is an explicitly given solution to the linear Black-Scholes equation without the PIDE part, i.e., $u^{B S}$ solves the linear parabolic equation:

$$
\frac{\partial u^{B S}}{\partial \tau}-\frac{\sigma^{2}}{2} \frac{\partial^{2} u^{B S}}{\partial x^{2}}-\left(r-\frac{\sigma^{2}}{2}\right) \frac{\partial u^{B S}}{\partial x}=0, \quad u^{B S}(0, x)=\Phi\left(K e^{x}\right), \tau \in(0, T), x \in \mathbb{R} .
$$

Recall that $u^{B S}(\tau, x)=K e^{x+r \tau} N\left(d_{1}\right)-K N\left(d_{2}\right)$ (call option case), where $d_{1,2}=(x+(r \pm$ $\left.\left.\sigma^{2} / 2\right) \tau\right) /(\sigma \sqrt{\tau})$ (c.f., [22,24]). Here, $N(d)=\frac{1}{\sqrt{2 \pi}} \int_{-\infty}^{d} e^{-\xi^{2} / 2} \mathrm{~d} \xi$ is the CDF of the normal distribution.

Theorem 2. Assume the transformed stock-trading strategy function $\psi(\tau, x)$ is globally $\omega$-Hölder continuous in both variables. Suppose that $v$ is a Lévy measure with the shape parameters $\alpha<3, D \in \mathbb{R}$, where either $\mu>0$, or $\mu=0$ and $D>1$. Let $X^{\gamma}$ be the space of Bessel potentials space $\mathscr{L}_{2 \gamma}^{p}(\mathbb{R})$, where $\frac{\alpha-1}{2 \omega}<\gamma<\frac{p+1}{2 p}$ and $\frac{1}{2} \leq \gamma<1$. Let $T>0$. Then, the linear PIDE (15) has a unique mild solution $u$ with the property that the difference $U=u-u^{B S}$ belongs to the space $C\left([0, T], X^{\gamma}\right)$.

Proof. First, we outline the idea of the proof. The initial condition $u(0, \cdot) \notin X^{\gamma}$ because of two reasons. It is not smooth for $x=0$, and it grows exponentially for $x \rightarrow \infty$ (call option) or $x \rightarrow-\infty$ (put option). The shifted function $U=u-u^{B S}$ satisfies $U(0, \cdot) \equiv 0$, and so the initial condition belongs to $X^{\gamma}$. On the other hand, the shift function $u^{B S}$ enters the 
governing PIDE as it includes the term $f\left(u^{B S}(\tau, \cdot)\right)$ in the right-hand side. Since $u^{B S}(0, x)$ is not sufficiently smooth for $x=0$, the shift term $f\left(u^{B S}(\tau, \cdot)\right)$ is singular for $\tau \rightarrow 0^{+}$. Following the ideas of [11], for the shift term $f\left(u^{B S}(\tau, \cdot)\right)$, we can provide Hölder estimates which are sufficient for proving the main result of this theorem (c.f., [11] Lemma 4.1). Furthermore, the exponential growth of the function $u^{B S}$ will be overcome since $\tilde{f}\left(e^{x}\right)=0$, where $\tilde{f}(u)=f(u)-\delta(\tau, \cdot) \partial_{x} u$, i.e.,

$$
\tilde{f}(u)(x)=\int_{\mathbb{R}}\left(u(x+\xi)-u(x)-\left(e^{\xi}-1\right) \partial_{x} u(x)\right) v(\mathrm{~d} z) .
$$

Next, we present more details of the proof. The function $u^{B S}$ solves the linear PDE (16). Thus, the difference $U=u-u^{B S}$ of a solution $u$ to (15) and $u^{B S}$ satisfies the PIDE with the right-hand side:

$$
\begin{aligned}
\frac{\partial U}{\partial \tau} & =\frac{\sigma^{2}}{2} \frac{\partial^{2} U}{\partial x^{2}}+\left(r-\frac{\sigma^{2}}{2}-\delta(\tau, x)\right) \frac{\partial U}{\partial x}+f(U)+f\left(u^{B S}\right)-\delta(\tau, x) \frac{\partial u^{B S}}{\partial x} \\
& =\frac{\sigma^{2}}{2} \frac{\partial^{2} U}{\partial x^{2}}+f(U)+g\left(\tau, x, \partial_{x} U\right)+h(\tau, \cdot),
\end{aligned}
$$

$U(0, x)=0, x \in \mathbb{R}, \tau \in(0, T)$. Here $g\left(\tau, x, \partial_{x} U\right)=\left(r-\sigma^{2} / 2-\delta(\tau, x)\right) \partial_{x} U$, and $h(\tau, \cdot)=\tilde{f}\left(u^{B S}(\tau, \cdot)\right)$. According to Proposition $2, f: X^{\gamma} \rightarrow X$ is a bounded linear mapping. Consequently, it is Lipschitz continuous, provided that $1 / 2 \leq \gamma<1$ and $\gamma>(\alpha-1) /(2 \omega)$. Clearly, $\tilde{f}\left(e^{x}\right)=0$. Hence,

$$
\tilde{f}\left(u^{B S}\right)=\tilde{f}\left(u^{B S}-K e^{r \tau+x}\right), \quad \text { and } \partial_{\tau} \tilde{f}\left(u^{B S}\right)=\tilde{f}\left(\partial_{\tau}\left(u^{B S}-K e^{r \tau+x}\right)\right) .
$$

Now, it follows from ([11] Lemma 4.1) that the following estimate holds true:

$$
\begin{gathered}
\left\|h\left(\tau_{1}, \cdot\right)-h\left(\tau_{2}, \cdot\right)\right\|_{L^{p}}=\left\|\tilde{f}\left(u^{B S}\left(\tau_{1}, \cdot\right)\right)-\tilde{f}\left(u^{B S}\left(\tau_{2}, \cdot\right)\right)\right\|_{L^{p}} \leq C_{0}\left|\tau_{1}-\tau_{2}\right|^{-\gamma+\frac{p+1}{2 p}}, \\
\|h(\tau, \cdot)\|_{L^{p}}=\left\|\tilde{f}\left(u^{B S}(\tau, \cdot)\right)\right\|_{L^{p}} \leq C_{0} \mid \tau^{-(2 \gamma-1)\left(\frac{1}{2}-\frac{1}{2 p}\right)},
\end{gathered}
$$

for any $0<\tau_{1}, \tau_{2}, \tau \leq T$. The function $h:[0, T] \rightarrow X \equiv L^{p}(\mathbb{R})$ is $((p+1) /(2 p)-\gamma)$ Hölder continuous because $\gamma<\frac{p+1}{2 p}$. Moreover,

$$
\int_{0}^{T}\|h(\tau, \cdot)\|_{L^{p}} d \tau=\int_{0}^{T}\left\|\tilde{f}\left(u^{B S}(\tau, \cdot)\right)\right\|_{L^{p}} d \tau \leq C_{0} \int_{0}^{T} \tau^{-(2 \gamma-1)\left(\frac{1}{2}-\frac{1}{2 p}\right)} d \tau<\infty,
$$

because $(2 \gamma-1)\left(\frac{1}{2}-\frac{1}{2 p}\right)<1$. We recall that the crucial part of the proof of ([11] Lemma 4.1) was based on the estimates:

$$
\left\|\tilde{f}\left(u^{B S}(\tau, \cdot)\right)\right\|_{L^{p}} \leq C_{0}\|v(\tau, \cdot)\|_{X^{\gamma-1 / 2}}, \quad \text { and }\left\|\partial_{\tau} \tilde{f}\left(u^{B S}(\tau, \cdot)\right)\right\|_{L^{p}} \leq C_{0}\left\|\partial_{\tau} v(\tau, \cdot)\right\|_{X^{\gamma-1 / 2}},
$$

where $v(\tau, x)=\partial_{x}\left(u^{B S}(\tau, x)-K e^{r \tau+x}\right)=K e^{r \tau+x}\left(N\left(d_{1}(\tau, x)\right)-1\right)$. This estimate is fulfilled because of Proposition 2 under the assumptions made on $\gamma$. The proof for the case of a put option is similar. The final estimate on the Hölder continuity of the mapping $h$ follows from careful estimates of the solution $u^{B S}$ derived in the proof of ([11] Lemma 4.1). The proof now follows from Theorem 1 and Proposition 3.

\section{Conclusions}

In this paper, we investigated the existence and uniqueness of a solution to the nonlocal nonlinear partial integro-differential equation (PIDE) arising from financial modeling. We considered a call/put option pricing model on underlying asset that follows a Lévy process with jumps in the multidimensional space. We employed the theory of abstract semilinear parabolic equation to obtain the existence and uniqueness of a solution to the PIDE in the scale of Bessel potential Sobolev spaces. We generalized existing results for a 
general Lévy measures that satisfies some suitable growth conditions. As an application to option pricing in one-dimensional space, we considered Black-Scholes models for pricing call and put options assuming that the asset follows a Lévy process. We obtained solutions of the governing nonlinear PIDE where the shift function depends on the large investor stock-trading strategy function, which is a solution to a nonlinear algebraic equation.

Author Contributions: The authors contributed equally to this research. All authors have read and agreed to the published version of the manuscript.

Funding: This research received no external support.

Institutional Review Board Statement: Not applicable.

Informed Consent Statement: Not applicable.

Data Availability Statement: Not applicable.

Acknowledgments: The authors gratefully acknowledge the contribution of the Slovak Research and Development Agency under the project APVV-20-0311 (D.Š.). The research was partially supported by the bilateral German-Slovakian DAAD Project ENANEFA (C.U.).

Conflicts of Interest: The authors declare no conflict of interest.

\section{References}

1. Abels, H.; Kassmann, M. The Cauchy problem and the martingale problem for integro-differential operators with non-smooth kernels. Osaka J. Math. 2009, 46, 661-683.

2. Florescu, I.; Mariani, M.C. Solutions to integro-differential parabolic problems arising in the pricing of financial options in a Lévy market. Electron. J. Differ. Equations (EJDE) 2010, 2010, 1-10. [CrossRef]

3. Aboodh, K.; Farah, R.; Almardy, I.; Almostafa, F. Solution of partial integro-differential equations by using Aboodh and double Aboodh transforms methods. Glob. J. Pure Appl. Math. 2016, 13, 4347-4360.

4. Arregui, I.; Salvador, B.; Ševčovič, D.; Vázquéz, C. Mathematical analysis of a nonlinear PDE model for European options with counterparty risk. Comptes Rendus Math. 2019, 357, 252-257. [CrossRef]

5. Mikulevičius, R.; Pragarauskas, H. On the Cauchy problem for certain integro-differential operators in Sobolev and Hölder spaces. Lith. Math. J. 1992, 32, 238-264. [CrossRef]

6. Ishii, H. Viscosity solutions of nonlinear partial differential equations. Sugaku Expo. 1996, 9, 135-152.

7. Burzoni, M.; Ignazio, V.; Reppen, A.M.; Soner, H.M. Viscosity solutions for controlled McKean-Vlasov jump-diffusions. SIAM J. Control Optim. 2020, 58, 1676-1699. [CrossRef]

8. Barles, G.; Buckdahn, R.; Pardoux, E. Backward stochastic differential equations and integral-partial differential equations. Stoch Int. J. Probab. Stoch. Process. 1997, 60, 57-83. [CrossRef]

9. SenGupta, I.; Mariani, M.C.; Amster, P. Solutions to integro-differential problems arising on pricing options in a Lévy market. Acta Appl. Math. 2012, 118, 237-249. [CrossRef]

10. Cont, R.; Voltchkova, E. Integro-differential equations for option prices in exponential Lévy models. Financ. Stoch. 2005, 9, 299-325. [CrossRef]

11. Cruz, J.; Ševčovič, D. On solutions of a partial integro-differential equation in Bessel potential spaces with applications in option pricing models. Jpn. J. Ind. Appl. Math. 2020, 37, 697-721. [CrossRef]

12. Cruz, J.; Ševčovič, D. Option Pricing in Illiquid Markets with Jumps. Appl. Math. Financ. 2018, 25, 389-409. [CrossRef]

13. Arregui, I.; Salvador, B.; Ševčovič, D.; Vázquéz, C. Total value adjustment for European options with two stochastic factors. Mathematical model, analysis and numerical simulation. Comput. Math. Appl. 2018, 76, 725-740. [CrossRef]

14. Henry, D. Geometric Theory of Semilinear Parabolic Equations; Lecture Notes in Mathematics; Springer: Berlin/Heidelberg, Germany; New York, NY, USA, 1981; Volume 840, p. iv+348.

15. Palatucci, G.; Kuusi, T. Recent Developments in Nonlocal Theory; De Gruyter Open: Warsaw, Poland, 2017.

16. Merton, R.C. Option pricing when underlying stock returns are discontinuous. J. Financ. Econ. 1976, 3, 125-144. [CrossRef]

17. Kou, S. A jump-diffusion model for option pricing. Manag. Sci. 2002, 48, 1086-1101. [CrossRef]

18. Madan, D.B.; Carr, P.; Chang, E.C. The Variance Gamma Process and Option Pricing. Eur. Financ. Rev. 1998, 2, 79-105. [CrossRef]

19. Barndorff-Nielsen, O.E.; Levendorski1̌, S.Z. Feller processes of normal inverse Gaussian type. Quant. Financ. 2001, 1, 318-331. [CrossRef]

20. Stein, E.M. Singular Integrals and Differentiability Properties of functions; Princeton Mathematical Series, No. 30; Princeton University Press: Princeton, NJ, USA, 1970; pp. xiv+290.

21. Brezis, H. Functional analysis, Sobolev spaces and partial differential equations; Springer Science \& Business Media: Berlin, Germany, 2010. 
22. Ševčovič, D.; Stehlíková, B.; Mikula, K. Analytical and Numerical Methods for Pricing Financial Derivatives; Nova Science Publishers, Inc.: Hauppauge, NY, USA, 2011; pp. 1-309.

23. Leland, H.E. Option pricing and replication with transactions costs. The journal of finance 1985, 40, 1283-1301. [CrossRef]

24. Kwok, Y.K. Mathematical Models of Financial Derivatives, 2nd ed.; Springer Finance; Springer: Berlin/Heidelberg, Germany; New York, NY, USA, 2008; p. xvi+530.

25. Avellaneda, M.; Levy, A.; Paras, A. Pricing and hedging derivative securities in markets with uncertain volatilities. Appl. Math. Financ. 1995, 2, 73-88. [CrossRef]

26. Ševčovič, D.; Žitňanská, M. Analysis of the nonlinear option pricing model under variable transaction costs. Asia-Pac. Financ. Mark. 2016, 23, 153-174. [CrossRef]

27. Schönbucher, P.J.; Wilmott, P. The feedback effect of hedging in illiquid markets. SIAM J. Appl. Math. 2000, 61, 232-272. [CrossRef]

28. Frey, R.; Stremme, A. Market volatility and feedback effects from dynamic hedging. Math. Financ. 1997, 7, 351-374. [CrossRef]

29. Frey, R.; Patie, P. Risk management for derivatives in illiquid markets: A simulation study. In Advances in finance and stochastics; Springer: Berlin/Heidelberg, Germany, 2002; pp. 137-159.

30. Jandačka, M.; Ševčovič, D. On the risk-adjusted pricing-methodology-based valuation of vanilla options and explanation of the volatility smile. J. Appl. Math. 2005, 2005, 235-258. [CrossRef]

31. Barles, G.; Soner, H.M. Option pricing with transaction costs and a nonlinear Black-Scholes equation. Finance and Stochastics 1998, 2, 369-397. [CrossRef]

32. Frey, R. Perfect option hedging for a large trader. Financ. Stochast 1998, 2, 305-325. [CrossRef] 